\title{
A novel mutated cell line with characteristics of dedifferentiated chondrosarcoma
}

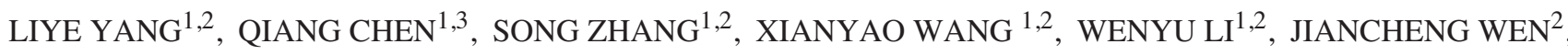 \\ XIAOPING HUANG ${ }^{2}$, JIAKUN ZHENG ${ }^{2}$, GE HUANG $^{1}$, TIANHUA HUANG ${ }^{1}$ and GUIZHI JU ${ }^{3}$ \\ ${ }^{1}$ Medical College of Shantou University, Shantou, Guangdong Province 515041; ${ }^{2}$ Department of Laboratory Medicine, \\ Chaozhou Central Hospital, No. 84 Huancheng Xi Road, Chaozhou, Guangdong Province 521021; \\ ${ }^{3}$ School of Public Health, Jilin University, Changchun 130023, P.R. China
}

Received April 16, 2009; Accepted June 15, 2009

DOI: 10.3892/ijmm_00000249

\begin{abstract}
Dedifferentiated chondrosarcoma (CS) is a rare, highly malignant variant of CS in which a high-grade sarcoma coexists with a low-grade chondroid tumor. In this study, a novel dedifferentiated CS cell line, MS0812, was spontaneously established from mutated human embryonic muscle cells. Several features of the cell line were investigated, including growth characteristics, cytogenetics, electron microscopic features, expression of various antigenic markers and tumor formation. MS0812 has been cultured continuously for more than 3 years. The growth characteristics of MS0812 are similar to the immortalized cell lines as reported. The cell line exhibited complex karyotypes and hyperploidy, the chromosome number ranged from 50 to 158 . MS0812 was positive for vimentin, desmin and muscle actin, indicating their muscle origin. With specific inductive condition, MS0812 differentiates into neural cells and adipocytes. Deletion of the $p 16$ gene, which seemed to play a major role in the malignant phenotype of this cell line, was confirmed by PCR and immunocytochemistry. MS0812 formed tumors in nude mice, and the tumor revealed a fibrosarcoma with chondroid components, which were consistent with dedifferentiated CS as reported. Chondroid components showed metachromasia by Alcian blue and toluidine blue and were S100 and collagen-II positive. To our knowledge, this is the first report of the establishment of a human dedifferentiated chondrosarcoma from mutated human embryonic muscle cells, and it is a useful model for the study of the molecular pathogenesis of dedifferentiated CS.
\end{abstract}

\section{Introduction}

Chondrosarcoma (CS) is the second most frequent primary malignant bone tumor after osteosarcoma and is characterized

Correspondence to: Dr Liye Yang, Medical College of Shantou University, Shantou, Guangdong Province, 515041, P.R. China E-mail: yangleeyee@sina.com

Key words: dedifferentiated chondrosarcoma, fibrosarcoma, chondrosarcoma, muscle stem cell, malignant transformation by basic neoplastic histology with cartilaginous stroma. The most frequent locations are the femur, pelvis and humerus, and $\sim 62 \%$ of patients are in the fourth, fifth and sixth decades of life (1-4). About $10 \%$ of CS undergoes regional anaplastic change, resulting in a high-grade noncartilaginous sarcoma arising within a typically low-grade CS, known as dedifferentiated CS (1-4), this concept was first introduced by Dahlin and Beabout in 1971 (5). They reported 33 cases of well-differentiated CS of bone with additional malignant mesenchymal components, such as fibrosarcoma or osteosarcoma. Some authors prefer instead to use the term CS with additional malignant mesenchymal component because of the morphogenesis of the tumor (5).

Dedifferentiated CS is a highly lethal malignancy. Despite adequate, aggressive, initial, wide surgical resection or even amputation, unfortunately, metastases occur early and frequently and commonly involve the lungs, lymph nodes, and viscera $(5-12)$. It is the poorly differentiated component that metastasizes. The prognosis is dismal, with an estimated 5-year survival rate of $<10-25 \%$ (5-12). Many analyses of dedifferentiated CS are available at the clinico-pathological level, and the cell biology of this neoplasm is still not clearly understood. To our knowledge, there is only one report describing the establishment of a cell line of dedifferentiated CS from a patient (13). We have established one such cell line, which was spontaneously transformed from human embryonic muscle-derived cells that may serve as a useful in vitro and in vivo model for study of the molecular pathogenesis of dedifferentiated CS.

\section{Materials and methods}

Sample source. An aborted human embryo, 10 weeks old, was collected following routine termination of pregnancy in conformity with the guidelines of China and appropriate informed consent of mother was signed. Embryo was from Chaozhou Central Hospital and processed within $1 \mathrm{~h}$.

The establishment of cell line and cloning. After extensive washing with sterile saline, sample was minced, and thigh muscle tissue was placed into sterile saline solution and was mechanically cut into $0.2-0.4-\mathrm{mm}^{3}$ pieces. The pieces were placed in a 12-cell plastic plate (Corning) containing DMEM/ $10 \% \mathrm{FBS}$ (GIBCO) medium with $2 \mathrm{mmol} / \mathrm{l}$ glutamine, $100 \mu \mathrm{g} / \mathrm{ml}$ 
penicillin, $100 \mu \mathrm{g} / \mathrm{ml}$ streptomycin and $2 \mu \mathrm{g} / \mathrm{ml}$ fluconazole, and the medium was just enough to cover the surface of the well and make the muscle pieces easily adhere to the plate. The plate was placed into an incubator, which was kept at $37^{\circ} \mathrm{C}$ in a humidified atmosphere with $5 \% \mathrm{CO}_{2}$. After incubation for $10 \mathrm{~h}$, enough culture medium was added. The culture medium was changed every 2 days, and when adherent cells began to proliferate and became confluent, cells were treated with $0.5 \%$ trypsin plus $0.2 \%$ EDTA for $5 \mathrm{~min}$. Enzyme activity was inhibited by adding DMEM plus $10 \%$ FBS and cells were centrifuged (600 x g, $10 \mathrm{~min}$, room temperature). Supernatants were discarded, cells were re-suspended in DMEM with $10 \%$ FBS, and plated into a flask $\left(5 \times 10^{3}\right.$ cells $\left./ \mathrm{cm}^{2}\right)$ in the same medium. The cells were passaged when they reached $70-85 \%$ confluence and cultured in the same way thereafter, and subcultured at a split ratio of 1:2 every 4-10 days.

When the cells were spontaneously transformed (named MS0812), they were diluted $\sim 10$ cells $/ \mathrm{ml}$, and were seeded into 96-cell plastic plates (Corning) in the same medium as above $(0.1 \mathrm{ml} /$ well $)$. Only wells with single cells were indicated, and when a single cell multiplied and became confluent, they were digested by $0.5 \%$ trypsin plus $0.2 \%$ EDTA as described above, and were seeded into a culture flask for propagation, monoclonal cell lines were selected and cultured for further study.

Transmission electronic microscopy. Transformed cells were digested, centrifuged and the sediment was fixed in $3 \%$ glutaraldehyde, buffered with $0.2 \mathrm{M}$ cacodylate at $4^{\circ} \mathrm{C}$, postfixed in $1 \%$ osmium tetroxide, dehydrated through ascending series of ethanol, and embedded in Epon 812. Semi-thin sections were cut and mounted on copper grids and stained with uranyl acetate and lead citrate, then examined under LEO 906 electron microscope.

Growth properties. Cells $\left(1 \times 10^{5}\right)$ were seeded in $35-\mathrm{mm}$ plastic dishes containing $2 \mathrm{ml}$ of DMEM supplemented with $10 \%$ FBS. At the indicated intervals, the cells were stained with $0.1 \%$ crystal violet in $0.1 \mathrm{M}$ citric acid, detached by trypsin digestion, and counted in a hemocytometer. Cell counts were performed every day for one week. Each data point represents the average of 3 dishes. The population doubling time was calculated from the log-phase growth curve.

For estimation of plating efficiency, 1,000 cells were seeded in $10-\mathrm{cm}$ plastic dishes containing $10 \mathrm{ml}$ of DMEM with $10 \%$ FBS, and were cultured for 14 days with a medium change on day 7 . Then the cultures were stained with $5 \%$ Giemsa in phosphate buffer to visualize colonies. The plating efficiency was determined by dividing the average number of colonies per dish by the number of inoculated cells per dish. Results are expressed as the mean of 3 dishes.

Chromosome analysis. The Standard trypsin-Giemsa banding technique (14) was performed for untransformed cells (passage 2, in vitro for 25 days) and MS0812 at the 100th passage. A total of 100 MS0812 cells with metaphase were analyzed.

Polymerase chain reaction (PCR). We used PCR to detect the expression of the p16 gene in MS0812 cells. Genomic DNA was isolated from MS0812 using a genomic DNA isolation kit (Universal Genomic DNA Extraction Kit Version 3.0,
Table I. PCR base sequence of the sense and antisense primers.

\begin{tabular}{llll}
\hline Primer nucleotide & ANT & Cycle & Size \\
\hline p16 exon 1 & & & \\
F1 (5'-cgg aga ggg gga gag cag gca-3') & $60^{\circ} \mathrm{C}$ & 35 & 277 \\
R1 (5'-gcg cta cct gat tcc aat tc-3') & & & \\
p16 exon 2 & & & \\
F2 (5'-ttc ctt tcc gtc atg ccg g-3') & $57^{\circ} \mathrm{C}$ & 35 & 394 \\
R2 (5'-gta caa att ctc aga tca tca gtc ctc-3') & & & \\
p16 exon 3 & & & \\
F3 (5'-ccc gct ttc gta gtt ttc at-3') & $58^{\circ} \mathrm{C}$ & 35 & 355 \\
R3 (5'-tta ttt gag ctt tgg ttc tg-3') & & & \\
B-actin & & & \\
F(5'-tgg cac cac acc ttc tac aat gag c-3') & $59^{\circ} \mathrm{C}$ & 35 & 396 \\
R(5'-gca cag ctt ctc ctt aat gtc acg c-3') & & & \\
\hline
\end{tabular}

PCR condition of each primer and product size, ANT, annealing time.

Takara Biotechnology Dalian Co., Ltd.) according to the manufacturer's instructions. The primers for PCR were synthesized in Shanghai Sangon Biological Engineering Technology \& Services Co., Ltd. PCR was performed for exons $1-3$ of the p16 (CDKN2A) in a KP-TC48 thermal cycler (Hybribio Ltd.). PCR was carried out in $10 \mathrm{mM}$ Tris (pH 8.6), $50 \mathrm{mM} \mathrm{KCl}, 1.5 \mathrm{mM} \mathrm{MgCl} \mathrm{m}_{2}, 0.4 \%$ DMSO, $0.2 \mathrm{mM}$ dNTPs, $0.4 \mathrm{mM}$ each primer, $50 \mathrm{ng}$ of genomic DNA, and 5 units of Taq polymerase (Takara Taq ${ }^{\mathrm{TM}}$, Takara Biotechnology Co., Ltd.) per $50 \mu 1$ reaction. The PCR conditions and primer sequences are noted in Table I. Primers for B-actin were used as a positive control for the presence of DNA. Electrophoresis of PCR products was performed on $2 \%$ agarose gel subsequently stained with ethidium bromide and photographed.

Adipogenic differentiation. MS0812 cells were digested and passaged as above, allowed to adhere for $24 \mathrm{~h}$ and culture medium was then replaced with specific inductive medium (15). For adipogenic differentiation, cells were cultured in DMEM plus 10\% FBS, $0.5 \mathrm{mmol} / 1$ 3-isobutyl-1-methylxanthine (Sigma), $0.5 \mathrm{mmol} / 1$ hydrocortisone (Sigma), $1 \mathrm{mmol} / 1$ dexamethasone (Sigma), $200 \mathrm{mmol} / \mathrm{l}$ indomethacin (Sigma), $10 \mu \mathrm{mol} / \mathrm{l}$ insulin (Sigma) and $50 \mu \mathrm{g} / \mathrm{ml}$ gentamicin for 2 weeks. Differentiated cell cultures were stained with Sudan black B (Sigma).

Neural differentiation. MS0812 cells were digested by trypsin from culture flasks and plated in $3.5 \mathrm{~cm}$ culture dishes, culture medium was refined with neural inductive medium. MS0812 were cultured in DMEM/F12 (1:1) plus N2 supplements (1:100, GIBCO), $20 \mathrm{ng} / \mathrm{ml}$ basic fibroblast growth factor (bFGF), $10 \mathrm{ng} / \mathrm{ml}$ epidermal growth factor (EGF) and $50 \mu \mathrm{g} / \mathrm{ml}$ gentamicin for 1 week. Differentiated cell cultures were fixed and stained with neural markers.

Tumorigenesis in nude mice. MS0812 cells grew logarithmically and reached confluence. Then they were digested by trypsin and EDTA from culture flasks and centrifugated. Cells were 

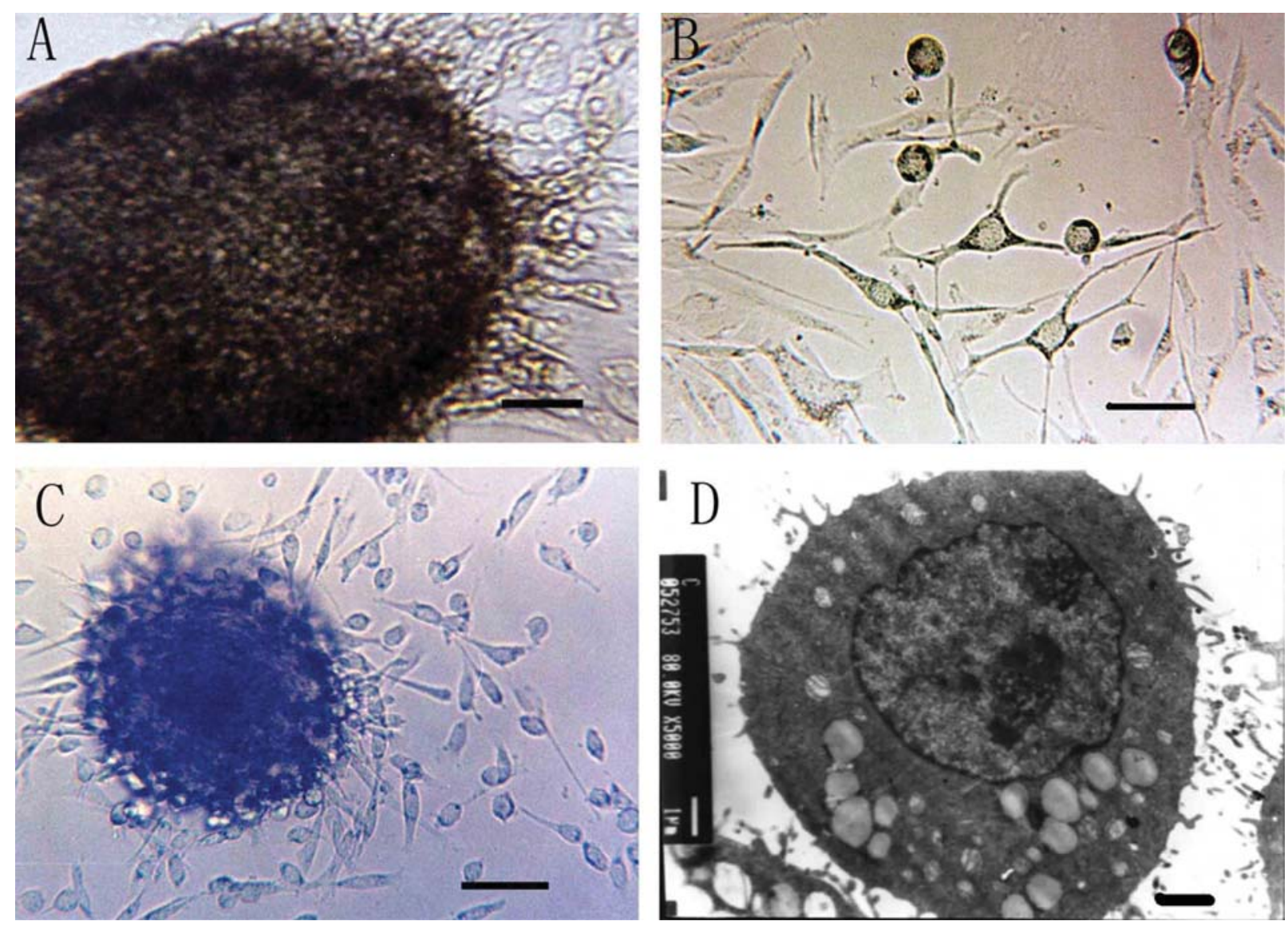

Figure 1. Morphological features of cultured cells. Cell migration from tissue pieces (A). A cell colony with rapid proliferation appeared, cells of interphase with round shape in the culture dishes (B). Morphological features of cultured MS0812 cells for long-term culture, they were spindle in shape, with oval nuclei, and cells lost contact inhibition, pile-up colony formed (C). Transmission electron micrograph of a tumor cell from the cell line MS0812. The nucleus showed two prominent nucleoli, and cell sphere shape uniformity with multiple microvilli, numerous vast cytoplasmic lipids in the cell were apparent (D). Scale bars in A and B, $100 \mu \mathrm{m} ; \mathrm{C}, 50 \mu \mathrm{m} ; \mathrm{D}, 1 \mu \mathrm{m}$.

diluted in Hank's solution. Tumorigenicity was examined by subcutaneous injection of $1-3 \times 10^{6}$ cells in $0.2 \mathrm{ml}$ of Hank's solution into the hind legs of nude mice (including male and female, 4-5 weeks old, from Experimental Animal Center of Sun Yet-sen University). Three months after tumor cell inoculation, the mice were sacrificed, tumor formation efficiencies were documented, and their livers, lungs, kidneys and brains were extracted for further analysis, local lymph node infiltration and distant metastasis were examined, and the collected tumors were cut, fixed in $10 \%$ neutralized buffered formalin, embedded in paraffin, sectioned in $5 \mu \mathrm{m}$ thickness, then were stained with hematoxylin and eosin (H\&E). Some samples were also selected for Alcian blue ( $\mathrm{pH} 0.5$ and $\mathrm{pH} 2.5$ ) or toluidine blue staining.

In two cases, inoculated tumors from nude mice were cultured and sub-cultured, and re-cultured cells were injected subcutaneously into second recipient nude mice to examine their tumorigenesis. Monoclonal cell lines from MS0812 were also injected subcutaneously into the backs of nude mice to examine tumor formation.

Immunostaining. Immunoperoxidase staining was performed both on formalin-fixed tissue sections and on cells prepared from the growing cultured cell lines.

The first antibodies were as follows: polyclonal rabbit anti-S100 (1/200 dilution); vimentin (monoclone V9, 1/200 dilution); desmin (monoclone 33, 1/100 dilution); PCNA (clone PC10, 1/100 dilution); muscle actin (clone HHF35, 1/100 dilution); $\alpha$-smooth muscle actin ( $\alpha$-SMA, clone A2547, 1/100 dilution); polyclonal rabbit anti-low and high molecular weight cytokeratins (AE1 and AE3, 1/100 dilution); polyclonal rabbit anti-myosin (1/100 dilution); MyoD1 (clone 5.2F, 1/100 dilution); p16 (clone 6H12, 1/100 dilution); p53 (clone DO7, 1/100 dilution); myf3 (clone3A11, 1/100 dilution); myogenin (clone F12B, 1/100 dilution); HLA-DR (clone LN3, 1/100 dilution); CD31 (clone1A10, 1/100 dilution); CD34 (clone QBEnd/10, 1/100 dilution); polyclonal rabbit anti-vascular endothelial growth factor c (VEGF-c, 1/100 dilution). All the above mentioned antibodies were from Zymed Laboratories Inc. South San Francisco, CA. Anti-collagen-II (monoclone 2B1.5, 1/200 dilution) antibody was from NeoMarkers (Fremont, CA); ß-III tubulin monoclonal antibody (1/2000 dilution) was from Sigma; anti-nestin was from Pharmingen of Canada (1/5000).

Samples were stained with the streptavidin-peroxidase complex kit (SP-9000, Beijing Zhongshan Corp.) as previously described (16). Diaminobenzidine (DAB) was used as the chromogen and hematoxylin as the nuclear counter stain.

For cell staining, cells were fixed with $4 \%$ paraformaldehyde (15 min), then were stained with the same method as previously described and 3-amino-9-ethylcarbazole (AEC) served as chromogen. 

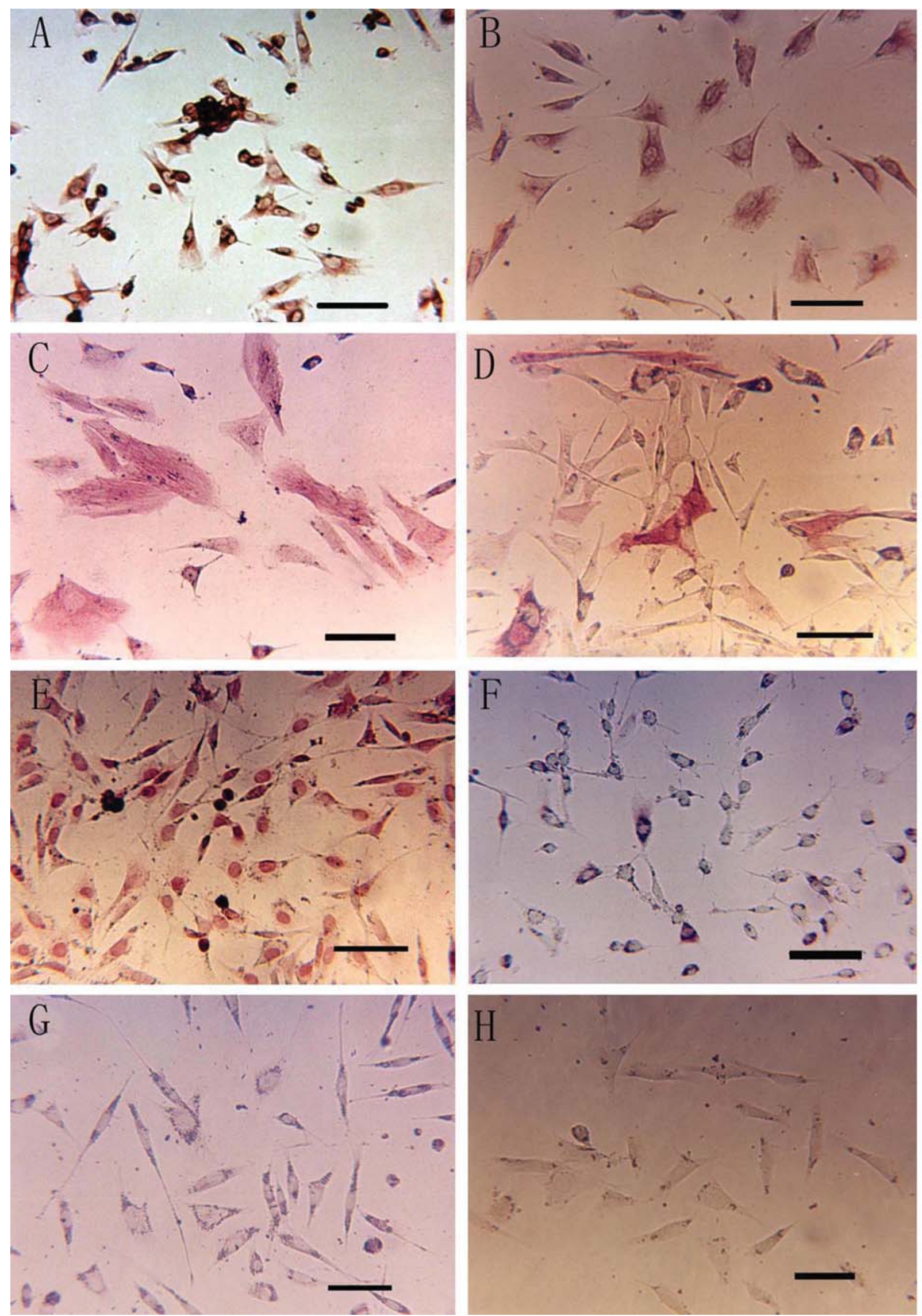

Figure 2. Immunostaining of myogenic and tumorous markers for MS0812. Positive staining for vimentin (A), desmin (B) and muscle actin (C). Positive staining for $\alpha$-SMA (D); positive staining for PCNA (E) and VEGF-c; Negative staining for p16 (G) and p53 (H). Scale bars in A, E, F, G, H and B, C, D, 100 and $50 \mu \mathrm{m}$, respectively. 


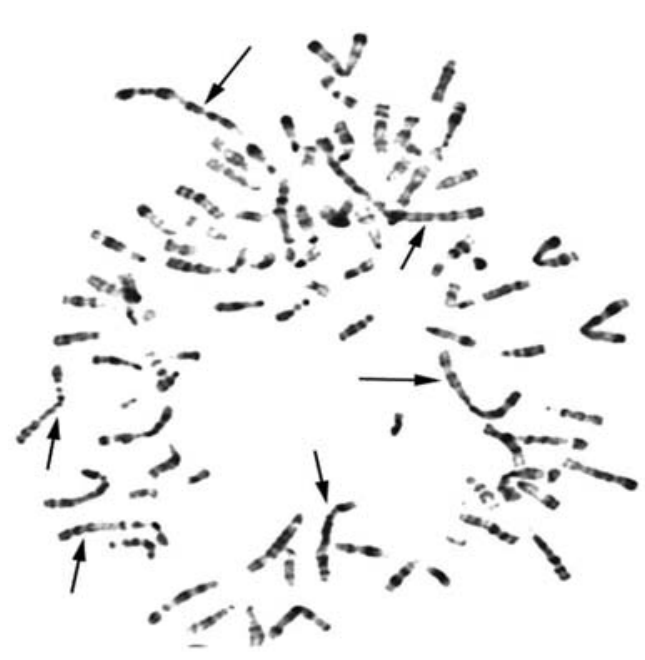

Figure 3. Karyotype of MS0812 showing apparent numerical and structural abnormalities (arrows)

\section{Results}

Morphological features of the cultured MS0812 cells. Initially, after 1-2 days of primary culture, cell migration was observed in culture tissue pieces, they presented spindle shape, with 1 round or ovoid nucleus under an inverted microscope. Adherent cells began to proliferate and became confluent after 10 days (Fig. 1A). After subculture, the cells looked like long spindleshaped fibroblastic cells, and began to form colonies and become confluent, $70-80 \%$ of primary cultured cells were myoblasts at this time, expressing MyoD1 and desmin, but not myosin (data not shown). After about 10 passages for 4 months in vitro, a cell colony of rapid proliferation appeared in the culture flask, the morphology of these cells changed significantly, metaphase was very common (Fig. 1B). After passage, the cells seeded onto plastic dishes began to proliferate immediately after adhering to the plastic surface without a noticeable lag period, they formed monolayer consisting of spindle cells (Fig. 1C), which, as they reached confluence, lost contact inhibition and became round and piled up, the cell line thus established was named MS0812, and has been maintained in vitro for $>120$ passages (a period of $>36$ months), and their characteristics are still retained. The population-doubling time of MS0812 cells in the logarithmic growth phase was $24 \mathrm{~h}$, and did not change at least up to 120 passages. In addition, plating efficiency of MS0812 cells was 128/1000 (12.80\%) on day 14 . The cell line grew well and adherent in a plastic dish with strong self-renewal capability, and multiplied rapidly.

Transmission electron microscopy revealed that the membrane surfaces of MS0812 cells were covered with abundant microvilli (Fig. 1D), the nucleus was enlarged, with round or irregular shape, one cell generally had one nucleus, 2 nuclei cells were discerned occasionally. Numerous lipid drops presence in the cytoplasm was the prominent feature of MS0812 cells (Fig. 1D), and no muscle fibre and transverse grain were identified in the cytoplasm of tumor cells.

MS0812 cells were analyzed for expression of a panel of cell markers by indirect immunostaining. They were positive for cytoplasmic immunoreactivity of vimentin (Fig. 2A) and desmin (Fig. 2B). Moreover, MS0812 cells were labeled for the expression of muscle actin (Fig. 2C) and $\alpha$-SMA (Fig. 2D).

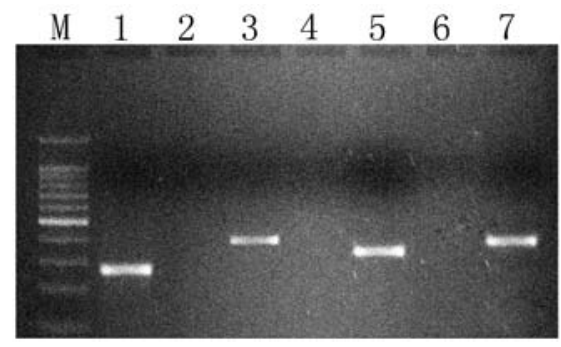

Figure 4. Homozygous deletion of the $p 16$ gene. Lanes from left to right, M, 100 bp ladder; 1 , exon 1 of normal control; 2, exon 1 of MS0812; 3, exon 2 of normal control, 4, exon 2 of MS0812; 5, exon 3 of normal control; 6, exon 3 of MS0812; 7, The internal control of $\beta$-actin of MS0812. Normal control is primary cultured human embryonic muscle cells.

In addition, positive staining for PCNA (Fig. 2E) and VEGF-c (Fig. 2F) were detected on the cultured cell line. They were not stained by p16 (Fig. 2G), p53 (Fig. 2H), CD31, myosin, cytokeratins, CD34, myosin, myf3, myogenin, S100 and HLA-DR (data not shown).

Transformed MS0812 cells were diluted $\sim 10$ cells $/ \mathrm{ml}$, then seeded into 96-cell plastic plates (Corning). Only wells with single cells were indicated, and we obtained 20 monoclonal cell lines from MS0812 for further study.

Karyotype characteristics. Cells of metaphase (100) were analyzed at the 100th passage. MS0812 cells showed a wide distribution of chromosome number and structural aberrations, with no distinct marker chromosome when examined; chromosome number was highly variable. All of the karyotyped cells had polyploidy characteristics, from 55 to 158 , cells with 71-81 accounted for $\sim 75 \%$ of all chromosomes, and apparent translocation was present in MS0812 cell (Fig. 3). The chromosome from untransformed cells of early passage in this donor was normal (data not shown).

p16 gene deletion. Homozygous deletions within 9p21-p22 was a small critical region of chromosomal loss in human malignant tumors. This region includes several classic tumor suppressor genes such as p16 (17). To assess the status of p16 in MS0812 cell lines, we used PCR assays in which $p 16$ exon 1, 2 and 3 fragments were amplified from genomic DNA. No p16 (including exon 1, 2 and 3) specific product was detected in MS0812. However, specific PCR products using genomic DNA from normal embryonic muscle cells were detected (Fig. 4).

Neural differentiation. MS0812 cells were digested by trypsin from culture flasks and plated in $3.5 \mathrm{~cm}$ culture dishes, and cultured in DMEM/F12 (1:1) plus N2 supplements, $20 \mathrm{ng} / \mathrm{ml}$ $\mathrm{bFGF}$ and $50 \mu \mathrm{g} / \mathrm{ml}$ gentamicin for 1 week. Neurogenic induction resulted in a change in MS0812 cell morphology, with almost all the cells assuming a neuron-like phenotype. Specifically, neuro-induced MS0812 cells underwent retraction, forming compact cell bodies with multiple extensions. Cell bodies became more spherical and cell processes exhibited branches with increasing induction time. A number of cells aggregated to form spheres. They showed hemi-floating state (Fig. 5A, B and C). Differentiated cell cultures were fixed and stained with neural markers, the majority of the cells were 

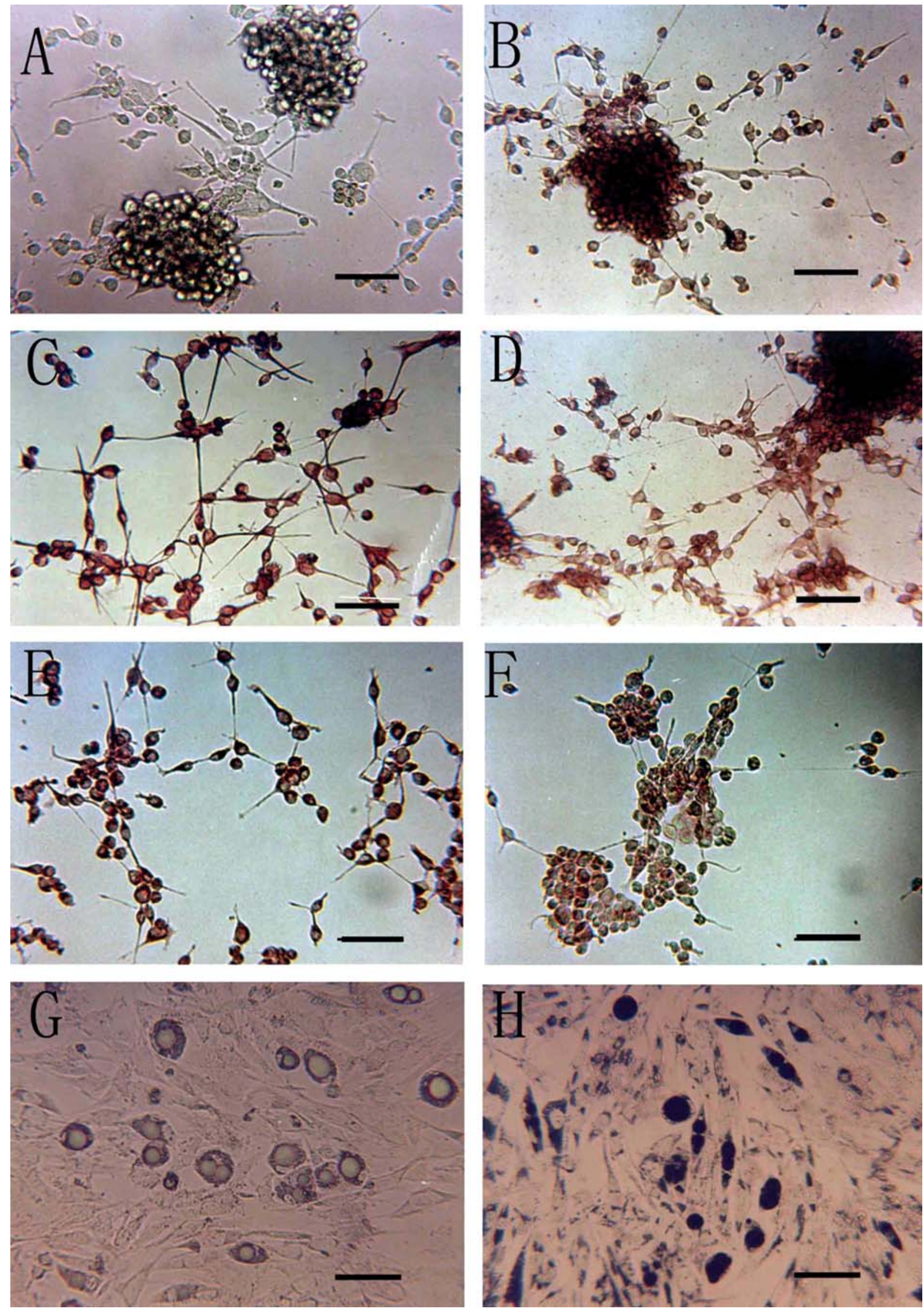

Figure 5. MS0812 differentiation into neural cells and adipocytes. Morphological change and immunostaining of MS0812 after neural induction (A, B, C, D, E, F). Cells were adherent on culture dishes or in suspension as aggregates after serum-free culture, similar to neurospheres of neural stem cells (A). Neuron-like cells from MS0812 were positive staining for nestin (B), the marker for neural stem cells, and also positive for B-III tubulin (C) and NF200 (D), the markers for neurons. Positive staining for GFAP (E) and S100 (F), the markers for glia. Morphological characteristics of MS0812 after adipogenic induction (G, H). Lipidscontaining adipocytes appeared on the culture dishes after adipogenic induction for 2 weeks $(\mathrm{G})$, and differentiated adipocytes were stained by Sudan black B (H). Scale bars in A, B, C, D, E, F and G, H represent 100 and $50 \mu \mathrm{m}$, respectively. 

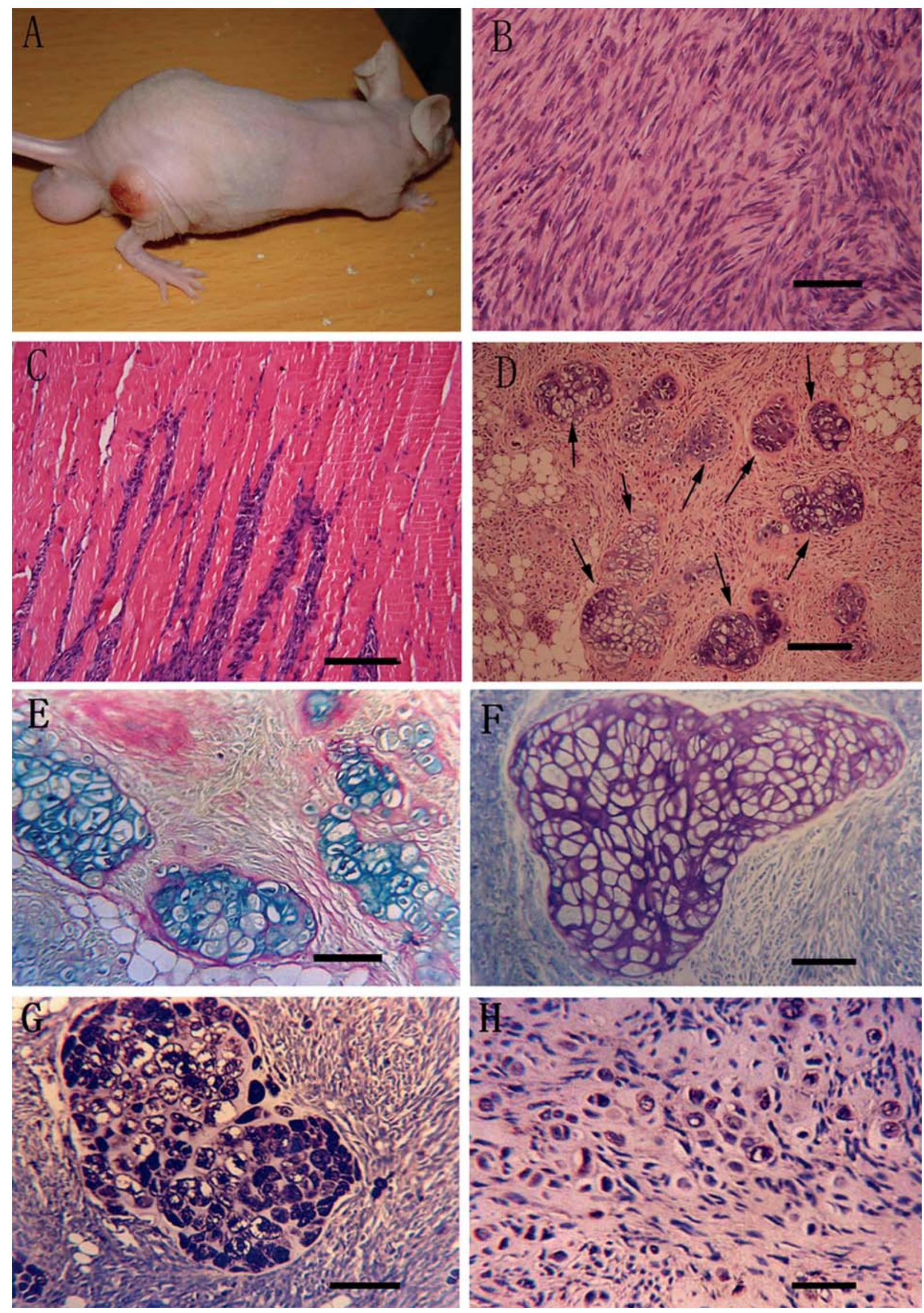

Figure 6. Tumor formation in nude mice. Photograph shows a tumor produced by injection of MS0812 cells into the subcutaneous tissue of the back leg of nude mice (A). The main components of tumors are malignant high-grade spindle cells (B) and tumor cells infiltrate into the muscle (C). Photomicrographs also show the tumor with a bimorphic pattern. The low-grade hyaline cartilage island (arrow) demonstrates the typical features of ordinary chondrosarcoma, surrounded by malignant high-grade spindle cells (D). Alcian blue staining demonstrates blue staining in the cartilage islands, suggesting the presence of proteoglycan (E). Chondroid cells from MS0812 show metachromasia when stained with toluidine blue (F). Immunohistochemically, cartilage islands were focally positive for S-100 (G), the chondroid cells reacted positively with collagenII (H). B, C and D are H\&E staining; A, B, C, D, and E, F, G, H represent 100 and $50 \mu$ m, respectively. 
positive for nestin (Fig. 5B), ß-III tubulin (Fig. 5C) and NF200 (Fig. 5D), consistent with the neuronal lineage. Surprisingly, they were also positive for GFAP and S100 (Fig. 5E and F), the markers for glia. These data show that MS0812 differentiates into cells of neural characteristics.

Adipogenic differentiation. MS0812 differentiated into lipid droplets containing cells under specific induction. The induction media for adipocyte is described above. After induction for 2 weeks, some cells differentiate into adipocytes, which contain lipid droplets (Fig. 5G). Sudan black B stained lipids droplets black (Fig. 5H). This shows that MS0812 possesses the ability of adipocyte differentiation. The cells of adipogenic differentiation account for $30-80 \%$ of the total cells in different tests. We performed the same experiments on 4 different monoclonal cell lines from MS0812. They also differentiated into droplets containing cells under the same induction media (data not shown).

Tumorigenesis and histopathology. MS0812 cells injected into nude mice and the small elastic hard nodules were first palpable in the mice 2-3 weeks after implantation with a formation rate of 14/14 (Fig. 6A). Three months later, the tumors had grown to $2.5 \mathrm{~cm}$ in diameters, the largest surgical sample measured $3.2 \mathrm{~cm}$ in diameter. The cut surface of these tumors were solid and the tumor consisted of soft, greyish-white tissue with foci of necrosis and small areas of hemorrhage. Long distance metastasis such as liver, lung, kidney, brain and lymph node was not identified. Multiple specimens of the tumor mass were fixed and embedded in paraffin. Sections $5 \mu \mathrm{m}$ thick were stained with $\mathrm{H} \& \mathrm{E}$. A histopathologic examination revealed that the malignant neoplasm was composed of two components. The main components of the tumor were predominantly storiform growth pattern or arranged in a compact bundle of markedly pleomorphic spindle cells with vesicular nuclei and one or more nucleoli, along with numerous mitoses, some of which were atypical, with pink cytoplasm (Fig. 6B). The tumor cells extensively invaded surrounding normal tissues such as dermis, muscular tissue (Fig. 6C), nerve and adipose tissues. The histologic features of the spindle cells closely resembled those of the fibrosarcoma and the other component was a low-grade chondrosarcoma, scattered in the form of islands, having a typical chondroid matrix with amitotic chondrocytes in lacunae, the nuclei showed moderate pleomorphism and hyperchromasia (Fig. 6D). Alcian blue stained the chondroid matrix blue (Fig. 6E), toluidine blue ( $\mathrm{pH} 4.1$ ) also stained the chondroid matrix purple (Fig. 6F). These findings correspond to differentiated CS.

Immunohistochemical studies showed that the tumor cells were diffusely positive for vimentin and PCNA, and the positive rate of PCNA was $\sim 100 \%$. The chondroid matrixes were focally positive for S100 and collagen-II (Fig. 6G and H), and malignant spindle cells predominantly negative for cytokeratins, S100, desmin, and $\alpha$-SMA (data not shown).

In two cases, tumors of MS0812 from nude mice were cultured and sub-cultured, and re-cultured cells were injected subcutaneously into second recipient nude mice and they also formed tumors.

We selected 10 monoclonal cell lines of MS0812 and injected the cells into the backs of nude mice. All monoclonal cell lines formed tumors in vivo, $9 / 10$ of them contained the components of CS and fibrosarcoma, especially in one case, where chondroid components constituted $80 \%$ of total tumor section, 1/10 clone was fibrosarcoma without cartilaginous tissue.

\section{Discussion}

Dedifferentiated CS was first proposed in 1971 by Dahlin and Beabout. They described a high-grade non-chondroid sarcoma, such as osteosarcoma, rhabdomyosarcoma, fibrosarcoma, and malignant fibrous histiocytoma (MFH), arising within a pre-existing low-grade CS (5). This dedifferentiated tumor represents $6-10 \%$ (1-6) of all CSs and frequently involves the extremities, especially the proximal femur and the pelvic bone. Histologic examination of dedifferentiated CS characteristically shows the presence of a well-differentiated CS juxtaposed to an anaplastic, non-chondroid sarcoma. The differentiated portion of the tumor consists most often of fibrosarcoma, as in this case, or MFH (2-5). The high-grade softtissue portions of the sarcoma usually comprise a larger volume of the tumor than the well-differentiated chondrosarcomatous areas (2-5).

In this study, we established an immortalized cell line (MS0812) from human embryonic muscle, this cell line has been cultured continuously for more than 3 years. MS0812 proliferated rapidly in vitro, and expressed PCNA, the marker for malignant cells, and formed tumors in vivo. The electron microscope showed the surface of cells has many microvilli. All these characteristics indicate their malignant features. We used a panel of antibodies to investigate the nature of tumor cells. The cell lines were positive for desmin, vimentin and muscle actin. Expression of these markers was indicative of muscle origin, and a number of MS0812 was also $\alpha$-SMA positive, indicating their myofibroblastic feature. Thus, we considered that MS0812 was possibly mutated from myoblasts (muscle stem cells) of human embryonic muscles, because myoblasts were desmin positive (15). Therefore, malignant transformation of myoblasts in vitro might contribute to the establishment of MS0812.

Immunohistochemical studies showed that the malignant spindle cells were strongly positive for vimentin, focally positive for S100 of chondroid sarcoma, and predominantly negative for cytokeratins, desmin, and $\alpha$-SMA. Taken together, the histologic and immunohistochemical findings were diagnostic of a dedifferentiated CS with fibrosarcoma as the predominant soft-tissue component. MS0812 showed tumor characteristics of fibrosarcoma and CS in vivo express phenotypes of neuron and adipocyte in vitro. Therefore, we considered that MS0812 possesses the characteristics of stem cells. These findings suggested that MS0812 is a novel tumor cell line with the capability of multiple differentiation. It may provide evidence for the theory that cancer originates from stem cells (18).

There is increasing evidence that the tumor suppressor gene mutations play a major role in the development, progression, and/or differentiation of tumors, with $p 53$ mutations and occurring frequently in the oncogenesis of various mesenchymal neoplasms (19-23). MS0812, in this case, did not show immunoreactivity for $p 53$, the reason for this needs further study. Recent genetic analysis revealed that p53 mutations were 
detected exclusively in dedifferentiated components. The evidence of a p53 mutation in the dedifferentiated component may be regarded as, at least, a cofactor that switches the preexisting low-grade chondrosarcoma to a highly malignant dedifferentiated tumor $(19,23)$. p16 deletion frequently occurred in multiple tumors, including sarcoma (17). MS0812 has a p16 deletion and results in a negative immunnohistochemistry with p16 staining. p16 and p53 seemed to play a major role in the malignant phenotype of this cell line.

At least three mechanisms for the origin of dedifferentiated CS have been hypothesized. The most popular theory is that the high-grade noncartilaginous component arises in a longstanding lower-grade CS (2-5). A second hypothesis is that the noncartilaginous sarcoma results from malignant transformation in an inflamed fibrous pseudocapsule of a lower grade CS. Finally, the third theory is the possibility that malignant cell lines arise simultaneously in a CS with differing ability to differentiate $(5,7,10,11,24-26)$. In our studies, monoclonal cell lines from MS0812 were able to give rise to $\mathrm{CS}$ and fibrosarcoma simultaneously in vivo. Based on clonal analysis, we concluded that CS and fibrosarcoma components of dedifferentiated CS originate from a monoclone with characteristics of multiple differentiation.

There are several reports on cell line establishment from CS (13,27-29). One was an immortalized cell line with characteristics of dedifferentiated CS (13). According to our knowledge, there is no immortalized cell line established from embryonic human muscle. We have established a mutated dedifferentiated CS from human embryonic muscles, and cell morphology, growth kinetics, and tumorigenesis characteristics were described. Our cell line is unique in that it showed the characteristics of muscle origin and multipotential differentiation in vitro, and only showed the characteristics of dedifferentiated $\mathrm{CS}$ in vivo. The cell line may therefore be a useful tool for investigating the biology and molecular target of treatment in dedifferentiated CS.

\section{Acknowledgements}

The authors thank Zheng Shuyan, Su Shaoxue and Liu Junbin for their technical assistance. This work was supported in part by the Natural Science Foundation of China (No.30672359) (to LY), Guangdong Province Social Development Plan (No. 2005B34001009) (to JZ) and China Postdoc Science Foundation (No.2004036182) (to LY).

\section{References}

1. Berend KR, Toth AP, Harrelson JM, Layfield LJ, Hey LA and Scully SP: Association between ratio of matrix metalloproteinase- 1 to tissue inhibitor of metalloproteinase-1 and local recurrence, metastasis, and survival in human chondrosarcoma. J Bone Joint Surg Am 80: 11-17, 1998.

2. Huvos AG: Chondrosarcoma including spindle-cell (dedifferentiated) and myxoid chondrosarcoma; mesenchymal chondrosarcoma. In: Bone Tumors. Huvos AG (ed). W.B. Saunders Company, Philadelphia, pp343-381, 1991.

3. Mirra JM and Marcove RC: Fibrosarcomatous dedifferentiation of primary and secondary chondrosarcoma. Review of five cases. J Bone Jt Surg Am 56: 285-296, 1974.

4. Unni KK: Chondrosarcoma. In: Dahlin's Bone Tumors. Unni KK (ed). Lippincott-Raven, Philadelphia, pp71-108, 1996.

5. Dahlin DC and Beabout JW: Dedifferentiation of low-grade chondrosarcomas. Cancer 28: 461-466, 1971.
6. Bruns J, Fiedler W, Werner M and Delling G: Dedifferentiated chondrosarcoma - a fatal disease. J Cancer Res Clin Oncol 131: 333-339, 2005.

7. Capanna R, Bertoni F, Bettelli G, Picci P, Bacchini P, Present D, Giunti A and Campanacci M: Dedifferentiated chondrosarcoma. J Bone Jt Surg Am 70: 60-69, 1988.

8. Staals EL, Bacchini P and Bertoni F: Dedifferentiated central chondrosarcoma. Cancer 106: 2682-2691, 2006.

9. Huvos AG: Chondrosarcoma including spindle-cell (dedifferentiated) and myxoid chondrosarcoma: mesenchymal chondrosarcoma. In: Bone Tumors: Diagnosis, Treatment, and Prognosis. Mitchell J (ed). 2nd Edition, W.B. Saunders Company, Philadelphia, pp343-393, 1991.

10. Frassica F, Unni K, Beabout J and Sim F: Dedifferentiated chondrosarcoma: a report of the clinicopathological features and treatment of seventy-eight cases. J Bone Joint Surg Am 68: 1197-1205, 1986.

11. Anract P, Tomeno B and Forest M: Dedifferentiated chondrosarcoma: a study of 13 clinical cases and review of the literature. Rev Chir Orthop Reparatrice Appar Mot 80: 669-680, 1994.

12. Mitchell AD, Ayoub K, Mangham DC, Grimer RJ, Carter SR and Tillman RM: Experience in the treatment of dedifferentiated chondrosarcoma. J Bone Joint Surg Br 82: 55-61, 2000.

13. Kudo N, Ogose A, Hotta T, Kawashima H, Gu W, Umezu H, Toyama $\mathrm{T}$ and Endo N: Establishment of novel human dedifferentiated chondrosarcoma cell line with osteoblastic differentiation. Virchows Arch 451: 691-699, 2007.

14. Seabright M: A rapid banding technique for human chromosomes. Lancet 2: 971-972, 1971

15. Pittenger MF, Mackay AM, Beck SC, Jaiswal RK, Douglas R, Mosca JD, Moorman MA, Simonetti DW, Craig S and Marshak DR: Multilineage potential of adult human mesenchymal stem cells. Science 284: 143-147, 1999.

16. Yang LY, Huang TH and Ma L: Bone marrow stromal cells express neural phenotypes in vitro and migrate in brain after transplantation in vivo. Biomed Environ Sci 19: 329-335, 2006.

17. Kamb A, Gruis NA, Weaver-Feldhaus J, Liu Q, Harshman K, Tavtigian SV, Stockert E, Day RS 3rd, Johnson BE and Skolnick MH: A cell cycle regulator potentially involved in genesis of many tumor types. Science 264: 436-440, 1994.

18. Li HC, Stoicov C, Rogers AB and Houghton J: Stem cells and cancer: evidence for bone marrow stem cells in epithelial cancers. World J Gastroenterol 12: 363-371, 2006.

19. Bovee JV, Cleton-Jansen AM, Rosenberg C, Taminiau AH, Cornelisse CJ and Hogendoorn PC: Molecular genetic characterization of both components of a dedifferentiated chondrosarcoma, with implications for its histogenesis. J Pathol 189: 454-462, 1999.

20. Grote HJ, Schneider-Stock R, Neumann W and Roessner A: Mutation of p53 with loss of heterozygosity in the osteosarcomatous component of a dedifferentiated chondrosarcoma. Virchows Arch 436: 494-497, 2000.

21. Nawa G, Ueda T, Mori S, Yoshikawa H, Fukuda H, Ishiguro S, Funai $\mathrm{H}$ and Uchida A: Prognostic significance of Ki67 (MIB1) proliferation index and p53 over-expression in chondrosarcomas. Int J Cancer 69: 86-91, 1996.

22. Oshiro Y, Chaturvedi V, Hayden D, Nazeer T, Johnson M, Johnston DA, Ordonez NG, Ayala AG and Czerniak B: Altered p53 is associated with aggressive behavior of chondrosarcoma: a long term follow-up study. Cancer 83: 2324-2234, 1998.

23. Simms WW, Ordonez NG, Johnston D, Ayala AG and Czerniak B: p53 expression in dedifferentiated chondrosarcoma. Cancer 76: 223-227, 1995.

24. Campanacci M, Bertoni F and Capanna R: Dedifferentiated chondrosarcomas. Ital J Orthop Traumatol 5: 331-341, 1979.

25. Daly PJ, Sim FH and Wold LE: Dedifferentiated chondrosarcoma of bone. Orthopedics 12: 763-767, 1989.

26. Mercuri M, Picci P, Campanacci L and Rulli E: Dedifferentiated chondrosarcoma. Skeletal Radiol 24: 409-416, 1995.

27. Kudawara I, Araki N, Myoui A, Kato Y, Uchida A and Yoshikawa H: New cell lines with chondrocytic phenotypes from human chondrosarcoma. Virchows Arch 444: 577-586, 2004.

28. Kunisada T, Miyazaki M, Mihara K, Gao C, Kawai A, Inoue H and Namba M: A new human chondrosarcoma cell line (OUMS-27) that maintains chondrocytic differentiation. Int $\mathbf{J}$ Cancer 77: 854-859, 1998.

29. Yagami K, Uyama Y, Yoshizawa Y, Kakuta S, Yamaguchi A and Nagumo M: A human chondrogenic cell line retains multipotency that differentiates into osteoblasts and adipocytes. Bone 34: 648-655, 2004. 